INPLASY

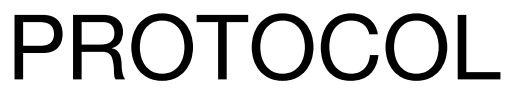

To cite: Yuan et al. Baduanjin on cancer-related fatigue for cancer patients: A protocol of systematic review and metaanalysis. Inplasy protocol 2020110139. doi:

10.37766/inplasy2020.11.0139

Received: 30 November 2020

Published: 30 November 2020

Corresponding author:

Qiang Yuan

yq1201986@163.com

Author Affiliation:

Hospital of Chengdu

University of Traditional

Chinese Medicine

Support: Chuan gan yan2020-513.

Review Stage at time of this submission: The review has not yet started.

Conflicts of interest:

The author has no conflicts of interest to declare.

\section{Baduanjin on cancer-related fatigue for cancer patients: A protocol of systematic review and meta-analysis}

Review question / Objective: Does Baduanjin intervention have ameliorative effects on cancer-related fatigue? Condition being studied: Cancer-related fatigue(CRF). Baduanjin.

Information sources: RCTS are being searched in the following electronic databases without language and publication date restrictions: PubMed, Cochrane Library, EMBASE, Web of Science, Scopus, PsycINFO, CINAHL, China National Knowledge Infrastructure (CNKI), WanFang Database, CQVIP and Chinese Biomedical Literature Data base (CBM) until Dec. 2020. Search terms are related to cancer related fatigue, Baduanjin.

INPLASY registration number: This protocol was registered with the International Platform of Registered Systematic Review and Meta-Analysis Protocols (INPLASY) on 30 November 2020 and was last updated on 20 December 2020 (registration number INPLASY2020110139).

\section{INTRODUCTION}

Review question / Objective: Does Baduanjin intervention have ameliorative effects on cancer-related fatigue?
Condition being studied: Cancer-related fatigue(CRF). Baduanjin

\section{METHODS}

Participant or population: Patients have confirmed diagnosis of cancer and with the 
symptom of CRF will be considered. There is no restriction on gender, age, types of cancer, tumor grade or post-treatment.

Intervention: Studies reporting the effects of Baduanjin intervention on multiple outcomes will be included if cancer-related fatigue is one of the outcomes. This review will include trials on Baduanjin or Baduanjin combined with other treatments; The control group, on the other hand, was waiting for treatment or other treatments. However, when the intervention in the treatment group was a combination of Baduanjin and other treatments, the corresponding control group should use the same other treatment. It does not limit the time, frequency and course of treatment of Baduanjin.

Comparator: A group of people with cancer-related fatigue symptom exposed to any other conventional or unconventional therapies apart from Baduanjin will be eligible as comparators. Exclusion: Other exercise as an intervention such as walking, jogging, Yoga will be excluded.

Study designs to be included: The study designs will include clinical RCTs of Baduanjin for CRF in cancer patients without any language or publication status restrictions. Non-RCTs, case reports, crossover studies, animal experiments and reviews will not be included.

Eligibility criteria: All adults aged $\geq 18$ years and with a diagnosis of cancer and with the symptom of CRF at any stage, type or phase of cancer treatment pathway.

Information sources: RCTS are being searched in the following electronic databases without language and publication date restrictions: PubMed, Cochrane Library, EMBASE, Web of Science, Scopus, PsycINFO, CINAHL, China National Knowledge Infrastructure (CNKI), WanFang Database, CQVIP and Chinese Biomedical Literature Data base (CBM) until Dec. 2020. Search terms are related to cancer related fatigue, Baduanjin.
Main outcome(s): Effectiveness of Baduanjin on Cancer-related Fatigue.

Additional outcome(s): Safety of Baduanjin on cancer-related fatigue.

Quality assessment / Risk of bias analysis: We will use the Cochrane Collaboration Risk of Bias Tool to evaluate the RCT design of these studies included in the review. Two reviewers will judge the risk of bias assessment for each study independently as high, low, or unclear, with discrepancies being resolved through discussion, and the third author being involved if necessary.

Strategy of data synthesis: Data will only be pooled if it is clinically meaningful and appropriate to do so. Otherwise, a narrative synthesis of the data will be conducted. Count data will be summarized using risk ratio (RR). Continuous data will be combined using mean difference with $95 \%$ confidence intervals (CI). If qualified studies shared the same scale, we will calculate mean difference (MD), otherwise standard mean difference (SMD) will be adopted. If the direction of CRF scale among studies are different (e.g. some scales had positive correlation with CRF while others were negative), the mean values measured by negative-correlated scale should multiply by -1 to ensure the consistency. Heterogeneity was tested by 12 test. If statistical heterogeneity is low $(12=50 \%)$, fixed-effects model will be adopted, otherwise random-effects model will be adopted (12>50\%). Then we will conduct sensitivity analyses based on study quality. All statistical analyses will be calculated in Review Manager 5.3 (the Cochrane Collaboration, Copenhagen, Denmark).

Subgroup analysis: If there is significant heterogeneity, we should firstly examine the causes and then carry out subgroup analysis. We will undertake subgroup analysis based on different diseases, different measurement tool and different intensity or follow-up period according to the details of included studies. 
Sensibility analysis: Based on sample size, study design, heterogeneous quality, methodological quality, and statistical model, sensitivity analysis will be performed to exclude trials with quality defects and ensure the stability of the analysis results.

Country(ies) involved: China.

Keywords: cancer-related fatigue; CRF; Baduanjin; cancer; protocol; systematic review.

Contributions of each author:

Author 1 - Qiang Yuan.

Author 2 - Xiaoyan Wang.

Email: 89042227@qq.com

Author 3 - Jianing Jian.

Author 4 - Qunwen Lu.

Author 5 - Jian Luo. 